\title{
Differential regulation of inflammatory mediators and granule-associated mediators in neonatal sepsis observed in cord and peripheral blood
}

This article was published in the following Dove Press journal:

International Journal of Interferon, Cytokine and Mediator Research

3 December 2013

Number of times this article has been viewed

\section{Raghunathan Muthukumar \\ Elden Berla Thangam}

Department of Biotechnology, School of Bioengineering, SRM University, Kattankulathur, Tamil Nadu, India
Correspondence: Raghunathan

Muthukumar

Department of Biotechnology, School

of BioEngineering, SRM University,

Kattankulathur, Kancheepuram (Dt),

Tamil Nadu, India 603203

Tel +9| 94427452270

Fax +9l 94427453903

Email muthukumarraghunathan44@gmail. com

\begin{abstract}
Neonatal sepsis is a systemic inflammatory response to bacterial infection. The innate immune system which acts as a first line of defense in infants, mounts a powerful inflammatory response upon pathogenic stimuli marked by elevated levels of inflammatory mediators and acute phase proteins, in the absence of a proper counteracting anti-inflammatory response. However, much less is known about the role of degranulation in development of systemic inflammation and sepsis. The present study reveals differentially regulated granular contents such as neutrophil elastase, nitric oxide, and myeloperoxidase which might act as key mediators in pathogenesis of neonatal sepsis. Neutrophil elastase and nitric oxide were found to be upregulated, while myeloperoxidase was found to be downregulated in neonatal sepsis. In addition, inflammatory mediators interleukin-6, interleukin-8, C-reactive protein, and procalcitonin were elevated in both cord blood and peripheral blood of septic neonates. These findings suggest that, in addition to inflammatory mediators, granule-associated mediators are also differentially regulated in neonatal sepsis and they might be used as sensitive and rapidly responding indicators of neonatal bacterial infection.
\end{abstract}

Keywords: systemic inflammation, acute phase proteins, C-reactive protein, neonatal spesis, myeloperoxidase, interleukin

\section{Introduction}

Neonatal sepsis is a highly inflammatory disease that culminates in septic shock and multiorgan failure due to uncontrolled activation of the inflammatory system in response to pathogens. ${ }^{1}$ The main causative organisms for neonatal sepsis include group B Streptococcus (GBS), Escherichia coli, Haemophilus influenzae, Listeria monocytogens, Staphylococcus aureus, and Klebsiella. ${ }^{2}$ Vertical transmission of bacterial infections from mother to infant contributes to early onset of infections. The late onset of sepsis on the other hand is usually attributed to environmentally transmitted infection. Defense against infection, especially in neonates with no immunological memory and low ability to develop specific antibodies against pathogens to which they are exposed during intrauterine and postpartum life, is predominantly dependent on innate immunity, which constitutes the first line of defense. ${ }^{3}$ The elements of innate immunity include neutrophils, monocytes, macrophages, dendritic and natural killer cells, and alternate complement system. Included in these neutrophils and macrophages are the primary immune effector cells at the site of inflammation that maintain the inflammatory response. ${ }^{4}$

The presence of pathogens is detected by the immune cells through germ lineencoded receptors such as Toll-like receptors, which recognize the pathogen-associated 
molecular patterns. A pathogenic stimulus triggers the mononuclear phagocytes to secrete various proinflammatory cytokines, which in turn promote the production of secondary inflammatory mediators by neutrophils. Soluble mediators like lysosomal enzymes and antimicrobial compounds are released by phagocytes and inflammatory cells for the clearance of pathogens. The microbicidal armory of neutrophils and macrophages consists of oxidative and non-oxidative processes that are activated simultaneously upon phagocytosis.

In addition to phagocytosis, neutrophils release preformed mediators that are stored in their granules, such as elastase, myeloperoxidase (MPO), and superoxide dismutase (SOD) by the process of degranulation. ${ }^{5}$ There is also production of a range of potentially damaging but ephemeral, reactive oxygen species (ROS) such as superoxide anion $\left(\mathrm{O}_{2}^{-}\right)$and reactive nitrogen species (RNS). ${ }^{6}$ The azurophilic granules of neutrophils largely contain antimicrobial peptides directed towards microbial killing and digestion, and they are the first to be reached at the site of infection. MPO, a major component of the azurophilic granules of neutrophils and monocytes, is essential for the oxygen-dependent microbicidal effect of neutrophils. ${ }^{7}$ All these compounds, including ROS, RNS, granular enzymes such as elastase, SOD, and MPO-derived oxidant hypochlorous acid contribute to polymorphonuclear (PMN) leukocyte/ macrophage-mediated killing of bacteria. An inflammatory response to pathogenic stimuli is crucial for host defense, which involves the regulation of both proinflammatory mediators and counteracting anti-inflammatory mediators. However, excessive production of proinflammatory mediators in sepsis overwhelms the anti-inflammatory signaling. While inflammatory responses are beneficial and protective at a moderate level, an excessive inflammation and acute phase response is detrimental, as it leads to tissue damage and organ dysfunction. ${ }^{8}$ The imbalance in the inflammatory network results in the suppression of the innate immune system (especially those of polymorphonuclear leukocytes) and leads to immunoparalysis and subsequently to an increased susceptibility to infection.

Hence, early diagnosis and treatment of infants with sepsis is a key to prevent life-threatening complications. Although extensive literature exists on single and combination laboratory tests, associated clinical signs, and risk factors to diagnose neonatal sepsis, the results have been conflicting, with each test having its own limitations. Postnatal diagnosis of sepsis is primarily limited by the requirement of peripheral blood of the neonate. The utilization of cord blood would be a suitable alternative, especially in diagnosis of early onset of sepsis.

The present study was aimed to examine the role of degranulation in development of systemic inflammatory response in neonatal sepsis by analyzing the levels of key mediators that are preformed in cytoplasmic granules of the immune cells (neutrophil elastase [NE], MPO, SOD, and nitric oxide [NO]) and also to analyze the proinflammatory mediators (interleukin [IL]-6 and IL-8) and acute phase reactants (C-reactive protein [CRP] and procalcitonin [PCT]).

\section{Materials and methods Sample collection}

The peripheral blood samples (from Neonatal Intensive Care Unit) and cord blood samples (from Labor Ward) were collected from SRM Hospital, Chennai, India, with the approval of the Institutional Ethical Committee and informed consent was obtained from the parent/guardian of all the subjects enrolled in this study. Cord blood samples were collected within 15 minutes of the delivery by squeezing and puncturing the cord using a clamp. Peripheral blood samples were obtained by vein puncture or drawn from a dwelling arterial or venous line. Both the samples were collected aseptically in collection tubes containing 3.8\% sodium citrate as anticoagulant. A total of 68 samples (which included 42 neonates with early onset sepsis and 26 healthy neonates) from both male and female neonates were collected.

\section{Isolation of plasma}

For plasma isolation, both the blood samples were centrifuged at $1,700 \times g$ for 20 minutes at $4^{\circ} \mathrm{C}$. The plasma was harvested and stored at $-20^{\circ} \mathrm{C}$ until further analysis.

\section{Estimation of various inflammatory mediators}

Estimation of levels of inflammatory mediators and granule-associated mediators in plasma was performed by fully automated assays carried out in duplicate according to manufacturer instructions for estimation of IL-6, IL-8 (eBioscience, San Diego, CA, USA), CRP (Diagnostics Biochem Canada Inc., London, ON, Canada), PCT (BRAHMS LIA, Hennigsdorf, Germany), NE (BioVEndor GmbH, Heidelberg, Germany), MPO (Calbiochem, Billerica, MA, USA), NO (Quantichrom, Hayward, CA, USA), and SOD (BioVision Inc., Milpitas, CA USA). The detection limits for the various assay procedures were $0.92 \mathrm{pg} / \mathrm{mL}$ for IL-6, $1.3 \mathrm{pg} / \mathrm{mL}$ for IL-8, $1.0 \mathrm{mg} / \mathrm{L}$ for CRP, $0.3 \mu \mathrm{g} / \mathrm{L}$ for PCT, $3.0 \mu \mathrm{g} / \mathrm{L}$ for $\mathrm{NE}, 0.1 \mu \mathrm{M} / \mathrm{L}$ for $\mathrm{NO}, 0.3 \mu \mathrm{g} / \mathrm{L}$ for $\mathrm{MPO}$, and $0.1 \mathrm{U} / \mathrm{mL}$ for SOD. PCT estimations were performed with the help of a luminometer (Berthold LB 952 [T16] luminometer, Hennigsdorf, Germany,) while all other estimations were performed with the help of a microplate reader (Multiskan Ex, Thermo Scientific, Waltham, MA, USA). 


\section{Statistical analysis}

The difference in estimated parameters between the neonates categories was analyzed by Student's $t$-test. All the parameters were analyzed at $95 \%$ confidence intervals, and a $P$-value of $<0.05$ was considered to be statistically significant. Statistical analysis of the data was performed using GraphPad Prism version 5.00 for Windows (GraphPad Software, San Diego, CA, USA).

\section{Results}

The present study involved a total of 42 neonates with early onset sepsis and 26 birth weight and gestational age matched healthy neonates. Cord blood was collected at the time of birth and was processed and stored, while peripheral blood samples were collected at the time of onset of clinical symptoms of sepsis and before initiation of any antibiotic therapy. Neonates were categorized as septic based on clinical symptoms, hematological parameters, and presence of bacterial infection. Clinical abnormalities observed in neonates with sepsis were abnormal body temperature (hypothermia and hyperthermia), temperature instability, respiratory distress such as grunting, cyanosis, lethargy, feeding intolerance, and diarrhea. Hematological abnormalities recorded in neonates with sepsis were abnormal total white blood cell count and abnormal levels of immature/total neutrophil counts. Antenatal risk factors observed in sepsis neonates were early onset of labor, rupture of membranes, vaginal discharge, and maternal fever. Neonates categorized as a sepsis group were further confirmed with high levels of CRP and PCT in blood.

\section{Levels of inflammatory mediators}

The inflammatory mediators IL-6, IL-8, PCT, and CRP were assessed in both cord blood and peripheral blood samples. The levels of IL-6, IL-8, PCT, and CRP at onset of clinical signs of sepsis are summarized in Table 1 for cord blood and Table 2 for peripheral blood. All the four inflammatory mediators were significantly upregulated in sepsis subjects as compared with normal neonates. The increased inflammatory mediators in sepsis samples were observed in both cord blood and peripheral blood.

\section{Levels of granule-associated mediators}

The lytic enzyme NE, the lysosomal peroxidase enzyme MPO, the antioxidant enzyme SOD, and the free radical NO were assessed in both cord blood and peripheral blood. The levels of NE, MPO, SOD, and NO at onset of clinical signs of sepsis are summarized in Table 1 for cord blood and
Table I Levels of inflammatory mediators and granule-associated mediators in cord blood

\begin{tabular}{lcc}
\hline Parameters & Control & Sepsis \\
\hline IL-6 $(\mathrm{pg} / \mathrm{mL})$ & $20.02 \pm 10.20$ & $264.1 \pm 29.8^{\mathrm{a}}$ \\
$\mathrm{IL}-8(\mathrm{pg} / \mathrm{mL})$ & $86.9 \pm 13.5$ & $630.9 \pm 70.4^{\mathrm{a}}$ \\
$\mathrm{CRP}(\mathrm{mg} / \mathrm{L})$ & $3.0 \pm 0.4$ & $6.7 \pm 0.8^{\mathrm{b}}$ \\
PCT $(\mu \mathrm{g} / \mathrm{L})$ & $0.40 \pm 0.02$ & $1.6 \pm 0.5^{\mathrm{a}}$ \\
Elastase $(\mu \mathrm{g} / \mathrm{L})$ & $229.0 \pm 14.6$ & $464.2 \pm 33.4^{\mathrm{a}}$ \\
NO $(\mu \mathrm{M} / \mathrm{L})$ & $23.6 \pm \mathrm{I}) \mathrm{I}$ & $42.2 \pm 2.8^{\mathrm{a}}$ \\
SOD activity $(\%)$ & $86.5 \pm 2.7$ & $63.1 \pm 3.7^{\mathrm{c}}$ \\
MPO $(\mu \mathrm{g} / \mathrm{L})$ & $24.1 \pm 8.5$ & $25.7 \pm 2.5$ \\
\hline
\end{tabular}

Notes: Values are expressed as mean \pm standard error of the mean. ${ }^{a} P<0.001$; ${ }^{\mathrm{b} P}<0.0$ I; ${ }^{\mathrm{c} P}<0.05$.

Abbreviations: CRP, C-reactive protein; IL, interleukin; MPO, myeloperoxidase; NO, nitric oxide; PCT, procalcitonin; SOD, superoxide dismutase.

Table 2 for peripheral blood. The granule-associated mediators $\mathrm{NE}$ and $\mathrm{NO}$ were significantly upregulated in both cord and peripheral blood of sepsis neonates as compared with healthy neonates. The level of MPO was downregulated in peripheral blood, while no change was observed in MPO levels in cord blood of sepsis neonates. SOD levels were downregulated in cord blood and upregulated in peripheral blood of sepsis neonates.

\section{Discussion}

Innate immune cells act as a first line of host defense, particularly neutrophils and macrophages, which on activation upon pathogen entry produce a number of proinflammatory cytokines and chemokines such as tumor necrosis factor (TNF)- $\alpha$, IL-1 $\beta$, IL-6, and IL-8, and other soluble mediators to eliminate the pathogens. The release of these mediators into the circulation results in the development of systemic inflammation in adults and infants. ${ }^{9}$ The proinflammatory cytokines and chemokines released by macrophages are potent neutrophil-activating agents. The resting neutrophils, which are primed with inflammatory agonists, get activated

Table $\mathbf{2}$ Levels of inflammatory mediators and granule-associated mediators in peripheral blood

\begin{tabular}{lcc}
\hline Parameters & Control & Sepsis \\
\hline IL-6 $(\mathrm{pg} / \mathrm{mL})$ & $19.7 \pm 7.7$ & $320.9 \pm 43.4^{\mathrm{a}}$ \\
IL-8 $(\mathrm{pg} / \mathrm{mL})$ & $73.6 \pm 17.7$ & $429.5 \pm 64 . \mathrm{I}^{\mathrm{a}}$ \\
CRP $(\mathrm{mg} / \mathrm{L})$ & $2.1 \pm 0.4$ & $5.4 \pm 0.7^{\mathrm{b}}$ \\
PCT $(\mu \mathrm{g} / \mathrm{L})$ & $0.5 \pm 0.1$ & $1.5 \pm 0.2^{\mathrm{a}}$ \\
Elastase $(\mu \mathrm{g} / \mathrm{L})$ & $142.0 \pm 8.7$ & $499.2 \pm 22.0^{\mathrm{a}}$ \\
NO $(\mu \mathrm{M} / \mathrm{L})$ & $31.0 \pm 0.2$ & $54.2 \pm 3 . \mathrm{I}^{\mathrm{a}}$ \\
SOD activity $(\%)$ & $35.0 \pm 1.4$ & $58.9 \pm 2.2^{\mathrm{a}}$ \\
MPO $(\mu \mathrm{g} / \mathrm{L})$ & $65.6 \pm 6.7$ & $21.2 \pm 3 . I^{\mathrm{a}}$ \\
\hline
\end{tabular}

Notes: Values are expressed as mean \pm standard error of the mean. ${ }^{a} p<0.00$ I; ${ }^{\mathrm{D} P}<0.01$.

Abbreviations: CRP, C-reactive protein; IL, interleukin; MPO, myeloperoxidase; NO, nitric oxide; PCT, procalcitonin; SOD, superoxide dismutase. 
and destroy pathogens by phagocytic and non-phagocytic processes. The phagocytes produce a number of toxic chemicals such as $\mathrm{O}_{2}, \mathrm{H}_{2} \mathrm{O}_{2}$, and hypochlorous acid that are not only involved in pathogen killing mechanisms but also contribute to host tissue damage. Upon pathogen invasion, these granular contents are released by degranulation for the clearance of pathogens. The present study assessed the levels of inflammatory and granule-associated mediators in cord blood and peripheral blood of sepsis neonates.

The study showed an upregulation of inflammatory mediators in both cord and peripheral blood of sepsis neonates. The results obtained were consistent with previous reports, where elevated IL-6, IL-8, PCT, and CRP in sepsis have been documented. ${ }^{10}$ IL- 6 and IL- 8 levels increase rapidly upon exposure to bacterial antigens and is usually known to precede increase in CRP. ${ }^{11}$ But IL- 6 and IL- 8 have very short half-lives, and their concentration drops to undetectable levels upon antimicrobial treatment. ${ }^{12}$ In most hospitals and primary health care centers in India, an indiscriminate antibiotic treatment is initiated based on clinical symptoms or antenatal risk factors, even before the time-consuming blood culture reports are made available to confirm the bacterial sepsis. The use of biological markers in addition to clinical assessment would increase the chance of precise diagnosis. Since the concentration of the cytokines may decrease upon time and antibacterial treatment, a multi biomarker approach would help in early detection of sepsis. Usually a combination of the cytokine assay determination with receptor expression or modified assay procedures to determine cytokine levels serve as better alternatives. ${ }^{12}$ In addition to the inflammatory mediators, incorporation of acute phase protein levels as indicators of bacterial sepsis would serve as a better alternative.

In newborn infants (premature as well as mature) with susceptibility to systemic infections, abnormal phagocyte functions have been reported. ${ }^{13}$ In this study, we have demonstrated that an elevated level of PMN-elastase was observed in both cord blood and peripheral blood of septic neonates as compared with normal. This reflects that although the concentration of PMN-elastase in healthy neonates is also reported to be higher during the first 3 days of life due to increased neutrophil numbers, ${ }^{14}$ significantly higher levels were found in neonatal systemic bacterial infection. Thus, our report is consistent with previous reports, and PMN-elastase is a sensitive and rapidly responding indicator of the neonatal bacterial infection, because it can be directly related to the presence of bacteria and is independent from the immunological system of the newborn infant. ${ }^{15}$
Several lines of evidence indicate that endogenously produced mediators such as $\mathrm{NO}$ and carbon monoxide are involved in the pathogenesis of sepsis. ${ }^{16}$ Previous studies confirm highly elevated NO concentration is related to the occurrence of septic shock and organ failure. ${ }^{17} \mathrm{We}$ also found elevated plasma NO levels in septic newborns in both peripheral blood as well as in cord blood. Infection and inflammatory insults lead to NO production by the inducible form of the enzyme (iNOS) found in macrophages, monocytes, neutrophils, and Kupffer cells. NO has a role in host defense by killing pathogens. Excessive levels, however, leads to pathologic loss of vascular tone, a central feature of sepsis-induced lethality ${ }^{18}$ and profound vasodilatation, hypotension, hyperactivity to catecholamines, and inadequate tissue perfusion. ${ }^{19}$

MPO, a heme enzyme present in the primary granules of PMN leukocytes, has been demonstrated to participate in the microbicidal activity of these cells. ${ }^{20}$ In this study, the peripheral blood MPO levels were found to be low in septic neonates compared to normal neonates, illustrating the presence immature immune system in neonatal sepsis. This MPO deficiency in PMN from neonates may contribute to their susceptibility to bacterial infection and systemic inflammatory state due to poor bacterial clearance. ${ }^{21}$ MPO has also been suggested to regulate the respiratory activity of PMN leukocytes during phagocytosis. During phagocytosis, PMN leukocytes undergo a burst in respiration and, through a series of single electron transfer, oxygen is reduced to $\mathrm{O}_{2}{ }^{-}$and $\mathrm{H}_{2} \mathrm{O}_{2}$. Although $\mathrm{H}_{2} \mathrm{O}_{2}$ alone is microbicidal, its bactericidal activity is greatly potentiated by the heme enzyme, MPO. Abnormal $\mathrm{O}_{2}$ consumption, formation, and $\mathrm{H}_{2} \mathrm{O}_{2}$ release have been reported by other researchers and interpreted as evidence for the impaired ability of MPO-deficient PMN leukocytes to terminate their respiratory burst. ${ }^{22}$

Oxidative stress occurs as a consequence of imbalance between the formation of oxygen free radicals and inactivation of these species by an antioxidant defense system. So, we also evaluated the level of SOD, which plays a key role in scavenging free radicals and thus limits oxidative injury in neonatal sepsis. In cord blood, the SOD level was low in septic samples when compared with normal samples. The reduced antioxidant capabilities as observed by low cord blood antioxidant levels, predisposes the neonate to sepsis. The body is unable to dispose of superoxide radicals and $\mathrm{H}_{2} \mathrm{O}_{2}$, which can attack all biological molecules and can cause tissue damage as reported in sepsis. ${ }^{23}$ We found increased concentrations of plasma SOD in septic patients compared with healthy controls, as reported in other data. ${ }^{24}$ 
The increased plasma concentrations of SOD in sepsis cases might be due to systemic inflammation and tissue damage, as is the case in several other clinical situations.

Our results suggest that there is a difference in production of inflammatory and granule-associated mediators by sepsis neonates as compared with that of healthy neonates. The granule-associated mediators NE and NO were found to be upregulated, which predispose neonates to a systemic inflammatory state and tissue damage, while downregulation of MPO accounts for poor bacterial clearance. In addition to inflammatory mediators, the granule-associated mediators also could serve as rapidly responding indicators of neonatal sepsis. Further analysis of other potent inflammatory mediators and their role in the development of systemic inflammatory response in neonatal sepsis is under investigation in our laboratory.

\section{Acknowledgments}

The authors are thankful to SRM University for financial assistance and to the Department of Biotechnology, Government of India for a research grant. The authors are also thankful to staff of SRM hospital for assistance in sample collection and to the research scholars of Integrative Medicine Laboratory, Department of Biotechnology for technical assistance.

\section{Disclosure}

The authors declare that they have no competing interests in this work.

\section{References}

1. Kenzel S, Henneke L. The innate immune system and its relevance to neonatal sepsis. Curr Opin Infect Dis. 2006;19:264-270.

2. Baltimore R, Huie SM, Meek JI, Schuchat A, Brien KLO. Early-onset neonatal sepsis in the era of group B streptococcal prevention. Pediatrics. 2001;108:1094-1098.

3. Pertova A, Metha R. Dysfunction of innate immunity and associated pathology in neonates. Indian J Pediatr. 2007;74:185-191.

4. Molloy EJ, Neill AJO, Grantham-Sloan JJ, Webb DW, Watson RWG. Maternal and neonatal lipopolysaccharide and Fas responses are altered by antenatal risk factors for sepsis. Clin Exper Immunol. 2008;151: 244-250.

5. Tung JP, Fraser JF, Wood P, Fung YL. Respiratory burst function of ovine neutrophils. BMC Immunol. 2009;10:1-11.
6. Sheppard FR, Kelher MR, Moore EJ, Mc Laughlin NJ, Banerjee A, Sillioman CC. Structural organization of the neutrophils NADPH oxidase: phosphorylation and translocation during priming and activation. J Leukoc Biol. 2005;78:1025-1042.

7. Kennedy AD, DeLeo FR. Neutrophil apoptosis and the resolution of infection. Immunol Res. 2009;43:25-61.

8. Cepinskas G, Wilson JX. Inflammatory Response in microvascular endothelium in sepsis: role of oxidants. $J$ Clin Biochem. 2008;42: $175-184$.

9. Medzhitov R, Janeway C Jr. Innate immunity. N Engl J Med. 2000;343: 338-344.

10. Mehr SS, Doyle LW, Rice GE, Vervaart P, Henschke P. Interleukin-6 and interleukin- 8 in newborn bacterial infection. Am J Perinatol. 2001;18:313-324.

11. Ng PC, Lam HS. Diagnostic markers for neonatal sepsis. Curr Opin Pediatr. 2006;18:125-131.

12. Orlikowsky TW, Neunhoeffer F, Goelz R, et al. Evaluation of IL-8concentrations in plasma and lysed EDTA-blood in healthy neonates and those with suspected early onset bacterial infection. Pediatr Res. 2004;56:804-809.

13. Miller E. Phagocytosis in the newborn infant: humoral and cellular factors. J Pediatr. 1969;74:255-259.

14. Manroe BL, Weinberg AG, Rosenfeld CR, Browne R. The neonatal blood count in health and disease. I. Reference values for neutrophils cells. J Pediatr. 1979;95:89-98.

15. Neumann S, Hennrich N, Gunzer G, Lang H. Enzyme-linked immunoassay for complexes of human granulocyte elastase with a1-p1 in plasma. In: Goldgerg DM, Werner M, editors. Progress in Clinical Enzymology, II. New York: Menson; 1983;37:270.

16. Terregino C, Lopez B, Karras D, Killian A, Arnold G. Endogenous mediators in emergency department patients with presumed sepsis: are levels associated with progression to severe sepsis and death. Ann Emerg Med. 2000;35:26-34.

17. Spack LP, Griffith HO. Measurement of total plasma nitrite and nitrate in pediatric patients with the systemic inflammatory response syndrome. Crit Care Med. 1997;25:1071-1078.

18. Bhagat K, Hingorani AD, Palacios M, Charles IG, Vallance P. Cytokineinduced venodilatation in humans in vivo: eNOS masquerading as iNOS. Cardiovasc Res. 1999;41:754-764.

19. Wolkow P. Involvement and dual effects of nitric oxide in septic shock. Inflamm Res. 1998;340:207-214.

20. Lau D, Mollnau H, Eiserich JP, et al. Myeloperoxidase mediates neutrophils activation by association with CD11b/CD18 integrins. Proc Natl Acad Sci U S A. 2005;102(2):431-436.

21. Grossl NA, Candel A, Shrit A, Schumacher HR. MPO deficiency and severe sepsis. Southern Medical Journal. 1993;86(7):832-885.

22. NauseefWM, Metcalf JA, Richard KR. Role of myeloperoxidase in the respiratory burst of human neutrophils. Blood. 1983;61(3):484-493.

23. Kumar A, Ranjan R, Basu S, Khanna HD, Bhargava V. Antioxidant levels in cord blood of low weight newborns. Ind Pediatr. 2008;45: 583-585.

24. Leff JA, Parson PE, Day CE, Tanuguchi N, Jochum M, Fritz H. Serum antioxidants as predictors of adult respiratory distress syndrome in patients with sepsis. Lancet. 1993;341:777-780.

International Journal of Interferon, Cytokine and Mediator Research

Dovepress

\section{Publish your work in this journal}

The International Journal of Interferon, Cytokine and Mediator Research is an international, peer-reviewed, open-access, online journal. The focus of the journal is to publish original research, reports, editorials, reviews and commentaries on all aspects of interferon, cytokine and mediators of inflammation from labora- tory science to therapeutic indications and clinical studies. The manuscript management system is completely online and includes a very quick and fair peer-review system, which is all easy to use. Visit http://www.dovepress.com/testimonials.php to read real quotes from published authors. 